Document downloaded from:

http://hdl.handle.net/10251/64698

This paper must be cited as:

Sendra, S.; Laborda, A.; Díaz Santos, JR.; Lloret, J. (2015). A Smart Bluetooth-based Ad Hoc Management System for Appliances in Home Environments. 13th International Conference, ADHOC-NOW 2014. Springer Verlag (Germany): LNCS. doi:10.1007/978-3319-07425-2_10.

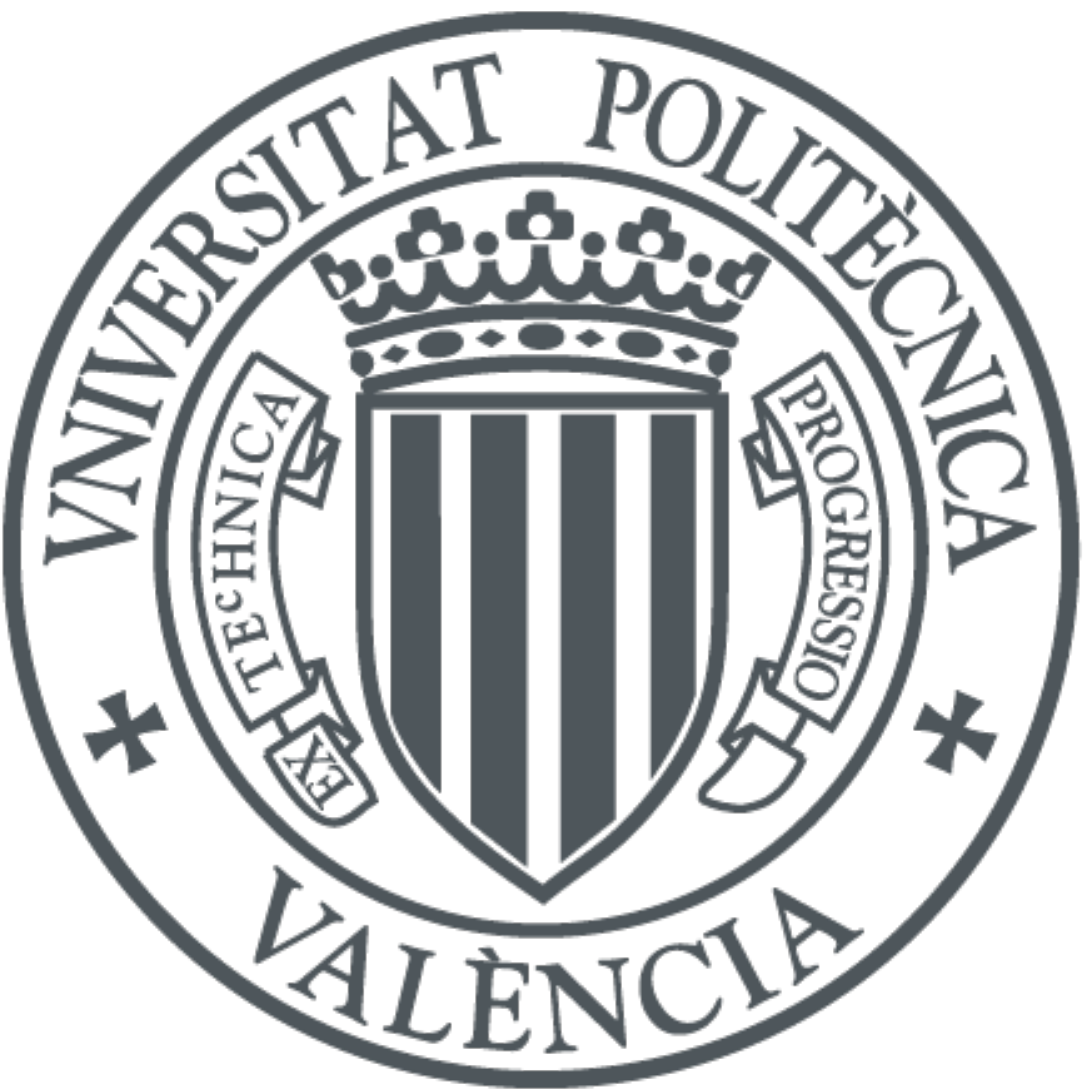

The final publication is available at

http://dx.doi.org/10.1007/978-3-319-07425-2_10

Copyright Springer Verlag (Germany): LNCS

Additional Information 


\title{
A Smart Bluetooth-based Ad Hoc Management System for Appliances in Home Environments
}

\author{
Sandra Sendra ${ }^{1}$, Antonio Laborda ${ }^{2}$, Juan R. Díaz ${ }^{1}$ and Jaime Lloret ${ }^{1}$ \\ ${ }^{1}$ Instituto de Investigación para la Gestión Integrada de zonas Costeras \\ Universidad Politécnica de Valencia \\ 46730 Grao de Gandia (Valencia), Spain \\ sansencolposgrado.upv.es, juadasan@dcom.upv.es, \\ lloretedcom. upv.es \\ ${ }^{2}$ Universidad SANJORGE \\ A-23 Zaragoza-Huesca Km. 299. \\ 50830 Villanueva de Gállego (Zaragoza), Spain \\ alu.23324@usj.es
}

\begin{abstract}
The number of home devices integrating new technologies is continuously increasing. These advances allow us to improve our daily routines. In addition, the improvement in network infrastructure and the development of smart phones and mobile devices allow us access from any place to any of our systems over the Internet. Bearing in mind this idea, we have developed a lowcost ad hoc protocol based on Bluetooth technology that allows us to control all our home appliances and monitor the power consumption of our homes. Our proposal is based on an Android application installed on a mobile device which acts as server. The application allows users to program the various appliances. It is also able to check the status of the appliance, as well as controlling the power consumption of the house and its cost. The system is equipped with a smart algorithm able to manage all appliances and decide which ones should work as a function of various criteria such as time of day or power consumption. Finally, the system is able to detect faults in water and electricity supply for acting accordingly. All data received and sent by the server are stored in a database which the system can check and compare to make their own decisions.
\end{abstract}

Keywords: Electrical household appliances; Bluetooth; Android applications; Smart Algorithm; Ad-hoc network, Internet of Things.

\section{Introduction}

The advances of new technologies in regards to electronic, communication infrastructures [1] and the Internet [2] in the last 10 years led us to think today would be living in smart homes [3]. However, automation is not yet implemented in most of our homes. Perhaps, we have only observed significant progress in management appliances, making home life similar to that of years ago. 
Currently, the smart appliances are eco-friendly, innovative and have very modern designs. This type of appliance can be managed using mobile phone applications. Thus, our smart phones/tablets become the control center from which we can remotely monitor our home.

The biggest technology brands like Panasonic, Samsung and LG, have available smart appliances suitable for domestic use and affordable for any user. These improvements allow a real time monitoring [4] of these smart appliances [5]. An example is The Smart Kitchen project [6]. It focuses on the usage of many small and inexpensive devices. Many small devices can be networked over a fieldbus in a domotic application. The advantage of fieldbus technology over other networking methods such as Ethernet is the high optimization level to the application.

There are several wireless technologies that our current mobile devices offer such as IrDA, Bluetooth, WiFi or NFC, among others. Bluetooth is the technology which presents the simplest mechanism for device discovering and their configuration. This technology also allows the implementing of ad-hoc networks and presents low power consumption. The prices of bluetooth devices are low. Its data transfer rate is enough for data exchange between smartphones and tablets since the expected traffic is small [7].

In addition, operating systems (OS) providers as Google has presented the new versions of its OSs (Jelly Bean of Android 4.3) which supports the new version of Bluetooth known as Bluetooth Smart (i.e. Bluetooth 4.0 on a dual chip). This technology minimizes the power consumption during the measurement process and data transmission. Therefore, devices such as tablets or smartphones using this version of Bluetooth present a more efficient use of battery. This new technology was already available in Apple from model iPhone 4S.

In this paper, we are going to present a new system to manage and control smartly our appliances at home. Our proposal includes, the mobile application developed using Android, the communication protocol used between our appliances and mobile devices and the smart systems used to intelligently decide which appliances should work in each moments as well as controlling the energy consumption at home.

The rest of the paper is structured as follows. Section 2 shows the background of the Smart application for controlling devices in home environments. Section 3 describes our proposed architecture for controlling our appliances and the applications developed to be installed in the different devices. The database used for storing all data is explained in Section 4, meanwhile Section 5 shows the communication protocol and the structure of each packet. Section 6 present the Android application developed for our system and the network tests. Finally, Section 7 shows the conclusion and future work.

\section{Related Work}

Because the great evolution of new technologies and advances in the field of Internet of Things (IoT), we can find several applications and systems developed for home environments. The main developed systems are assistive environment applications and body area networks [8][9], healthcare [10], behavior monitoring systems [11], 
passive localization and people tracking and power metering [12], among others. Other applications, in regards to smart homes, are the intelligent management of appliances in home environments. In this section, we are going to present some smart applications developed for controlling different electronic devices in home environments.

J. Lloret et al. presented in [13] a low-cost communication architecture that increases the safety and quality of life at home. The proposed architecture allows sensors, which detects physical parameter changes in household appliances to be managed via Twitter. Authors proposed a smart system able to gather data from sensors. The information from sensors is fused in order to notify the user by messages when some event has happened in order to take decisions.

A. Kamilaris [14] presented an application framework with concurrent, multi-user support that facilitates the development of advanced ubiquitous applications by habitants without programming experience to automate his house. Authors resume the main features and requirements that future Smart Homes should present. These systems should be open and accessible for simultaneous users for interacting directly with sensors and actuators, and they should allow continuous monitoring. They also mplemented a small number of RESTful Web Services in TinyOS and performed several test to define the application framework performance and the device discovery timing.

Finally, A. Kamilaris et al. also presented [15] a system that includes a 6LoWPANbased wireless sensor network inside the home environment. Author compensate the overhead embedding the IPv6 stack on sensor devices by using HTTP caching to reduce the mean response time to access sensor data. Finally, authors developed a graphical interface to abstract home automation procedure for typical home residents. Through the different tests and technical evaluation, the system show several benefits enabling embedded sensors in terms of performance and energy conservation.

As we can see, there are lots of possibilities and developments to improve our daily live. Our proposal also tries to make easier our routines and chores. This system allows us the monitoring of our entire house including the activity of the different appliances and energy consumption monitoring in real-time.

\section{Proposed Architecture and Control Applications}

In this section, we are going to present our proposed architecture for controlling our appliances. Our system is based on a distributed communication architecture which allows us the fast communication and execution of actions on each appliance. We will also show the operation diagram for client and server and the smart system which will inform us about the power energy consumption at home and the current price as a function of our service provider and the kind of service.

The proposed architecture is based on a server (smartphone or tablet) and other smart appliances that are indoors. All of them are equipped with Bluetooth cards. Actions on each device are performed in a distributed manner.

Our network presents a decentralized topology where the server is located on the center of network and the appliances are the intermediate nodes. The server has 
embedded a database (generated using MySQL) which keeps information of the different elements to be managed.

We can find two different scenarios. On the one hand, all appliances and server are within the house. So, the server performs a local access to different devices (see Figure 1). The server collects the status of each appliance including the electric power consumption. The server queries the price of KWh as a function of the type of services and the smartphone shows in its screen the current value (in Euros) of the consumed energy. In this case, the server and appliances should be in the coverage area in order to communicate all nodes. If we would like to spread signal along the house, we should implement a scatternet. A scatternet is a number of interconnected piconets that supports communication between more than 8 devices [16].

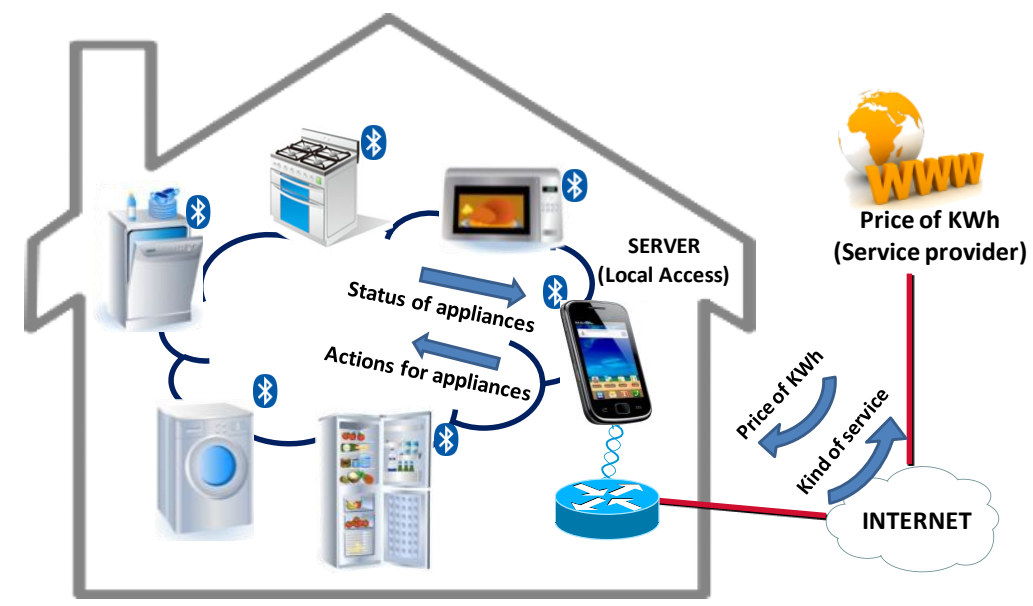

Fig. 1 Architecture for local access

As Figure 2 shows, the second situation places the server outside the home. It means that access to appliances will be remote. When the network does not detect the presence of the smartphone, a computer will act as local alternative server. This local server will act as a gateway between Bluetooth home network and the Internet. It is also able to store all events that happens in the house. When smartphone is in the Bluetooth network, the local alternative server will send this information to the smartphone.

The goal of eliminating the alternate server in the presence of the smart phone is to reduce the power consumption at home. The logic that manages the communication between the server and the various devices is performed by a mobile application (App) developed on Android. In addition, the specific software to interact with Bluetooth card devices is developed in Java.

Because we are using Bluetooth technology, the possible problem of nodes synchronization is solved by the characteristics of the standard where the system is synchronized using a global clock and a specific pattern of jumps, both unique. In our case, our master device provides the synchronization reference from its internal clock and other devices operate as slaves. 


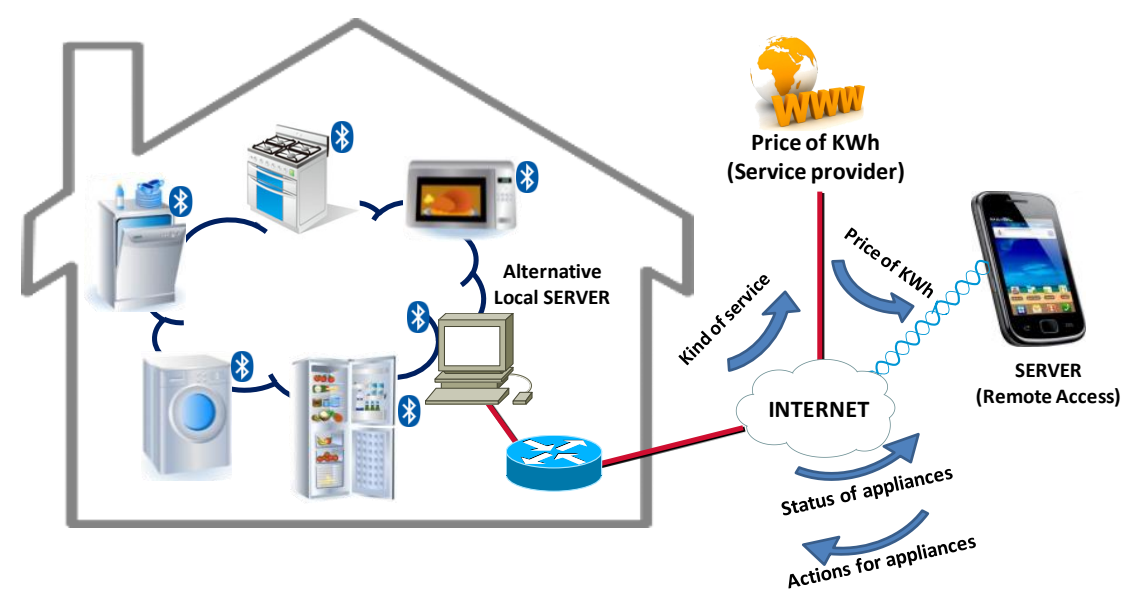

Fig. 2 Architecture for remote access.

\subsection{Control Application for Server}

Our control application is able to communicate with any device connected to it. There exist different types of orders and actions to be sent to the devices. Thus, the user can interact with a single device by a specific order or send a global command to turn off all appliances, if there is any fault or serious incident.

The first step of control application is to register the time and check that there is no problem with water and energy supply. If an appliance such as washing machine is running and the water supply has been cut off, the device will probably break. If there is an outage of light, the control application will store the program that had the appliance and the appliance state where it left off.

For normal operation where there are no problems in energy/water supply, the application will register which appliances are scheduled to work during the day. Depending on the time of day, the system will decide which appliances should work.

These decisions can be configured by the user according to different criteria. The first one can depend on the energy consumption. The user can, e.g., specify the system that the dishwasher and the washing machine perform their functions at night to use the reduced prices rates.

Another criterion may be due to user preferences such as oven cook food in the hours near to lunch time instead of doing laundry. Thus, the user could maintain constant energy consumption in their homes.

After the training phase, the algorithm will analyze each received data which will be compared with the data from the database. After determining the priority of appliances and a possible alarm, the system will perform, on the one hand, a tagging and storing of the appliances priority and alarm, on the other hand, the communication to the device. Figure 3 shows the flow diagram for control application. 


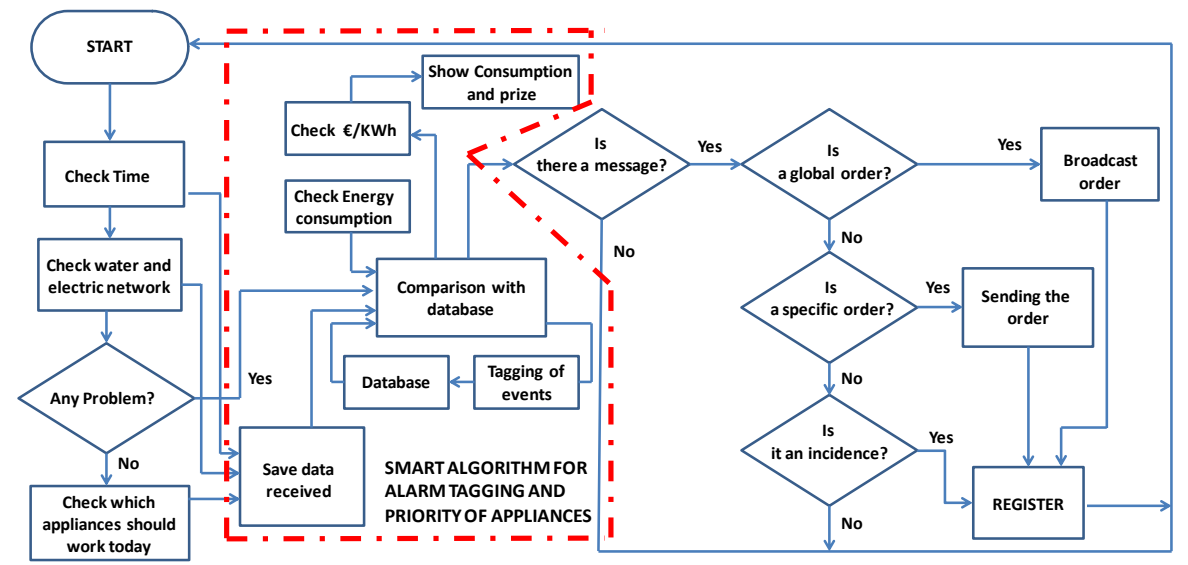

Fig. 3 Flow diagram of smart algorithm for control application.

\subsection{Control Application for Client}

For each device, an application for actions managing is implemented. Figure 4 depicts the flowchart of the client application. The client application installed on each device analyzes what type of order has received (global or specific) and the origin of this order (from user or from the device itself).

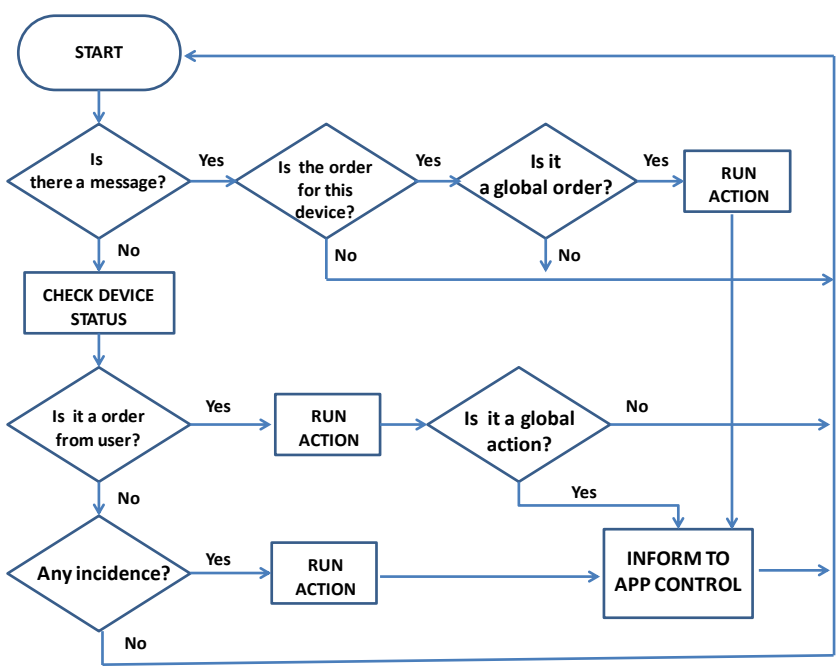

Fig. 4 Flow diagram for client app.

In this way, each Bluetooth card will act as its own master. But the control application will take the most important role. It concentrates the intelligence of system and it will be responsible for analyzing the environment, time and appliances that must work today. After the learning period where the user has made a regular use 
of their appliances, the system will be able to decide what appliances should work depending on the time of day. However, each device will be able to make their own decisions about how to improve their situation.

Decentralizing part of decisions on the actions of the device allows us to have a more reliable fault-tolerant system. If an error occurs in the control application or in the communications between the control application and the different appliances, each device may act separately, considering a series of actions based on the last recorded state. In addition, the information flow will be distributed in whole system.

\section{Database Structure}

This section explains the database structure used to manage and store the information.

In order to manage the flow of information between the device and the App control, it is needed a repository where performing queries. This repository or database can be easily developed using SQL statements. Our database will store information about data from each device, their programming and programs, their faults, the different commands sent to the devices as well as the status of programming and solution for a given fault.

The information of each device is structured and stored in tables and the relationships between each field are shown in Figure 5.

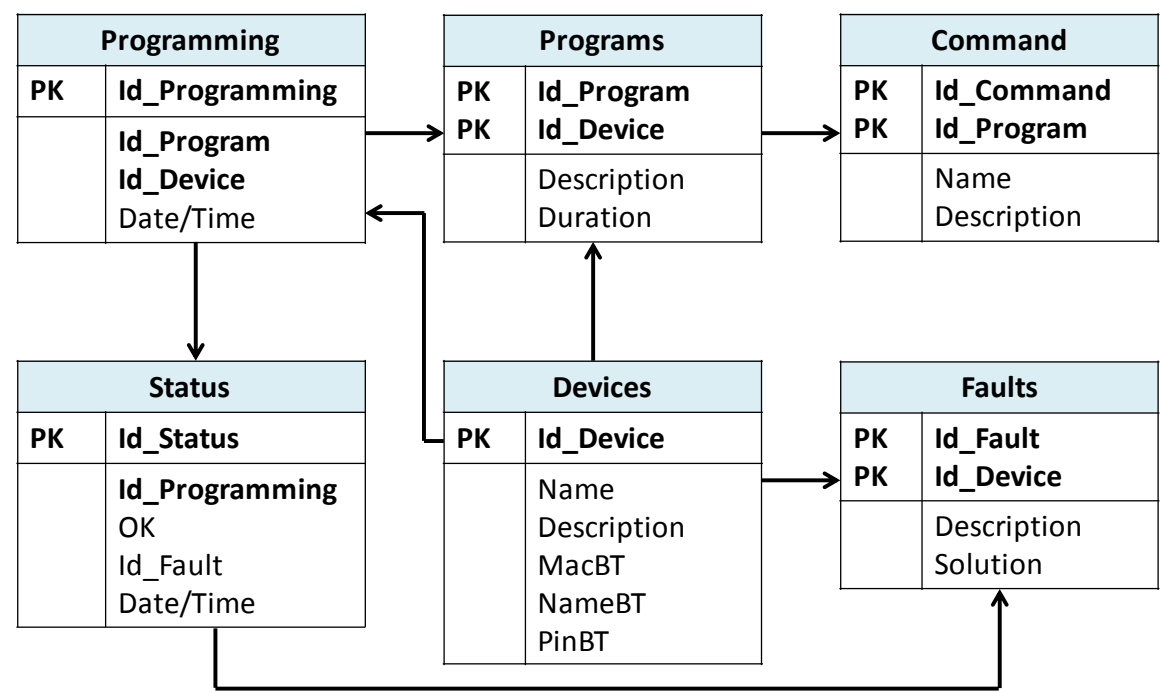

Fig. 5 Design and structure of database

The names given to each field and tables are representative of its content. Table "Programs" stores the description and duration of a particular program for a device. Table "Faults" stores a description a fault and a possible solution for a device. Table "Programming" stores for a device and its program, the date and time of the different programs that we have made. Table "Status" stores for a determined Programming, 
the status of device within its program, the status of a possible solution, and the date and time of each event. The "Id_Fault" field in "Status" table stores a fault code generated for an appliance. The system tries to find a solution as a function of this fault code. The server sends a set of orders to the appliance accordingly to the available information within the field "Solution". The Date field displays the last programming updated in the status field of that device. Table "Devices" store a full description of each device. Finally, Table "Command" stored the set of commands that correspond to a determined program.

\section{Communication Protocol}

In order to communicate all appliances and control application, it is needed to design a communication protocol. Our proposal is an open protocol. It is very basic and simple but very effective. This section shows the different kinds of packets we have. Each packet identifies the different fields it has.

When we need to design a communication protocol for applications monitoring, it can be use closed protocols or owners of a particular brand. Sometimes these protocols may be variants of standard protocols. However, this fact will limit us to use devices from a particular brand. We may also use open protocols defined between several companies in order to unify criteria. Usually, there are not patents related on this protocol. So, any manufacturer can develop applications and products which carry this communication protocol. Some examples of the most widely used open protocols are KNX [17], Lonworks [18], X10 [19], among others.

For our application, we have designed an easy protocol that defines three types of packets. The first one shows information about settings that server can send to the appliances. Figure 6 shows the structure of programming packet. The second packet (See Figure 7) is in charge of reporting the status of program for a given appliance, from the appliance to the server. Finally the third packet allows us managing any faults that may occur on a specific appliance. The way you manage faults is very simple. If a fault code is received, the server will query the database for possible solutions and after that, it will send the code of solution to the appliance (see Figure 8).

\begin{tabular}{|l|l|l|l|l|l|l|l|l|}
\hline Id_Programming @ Id_Device $@$ MacBT & Id_Program $@$ Id_Comand $@$ Date/Time \\
\hline
\end{tabular}

Fig. 6 Structure of programming packet.

\begin{tabular}{|c|c|c|c|}
\hline Id_Programming & @ & Id_Status & $@$ \\
\hline
\end{tabular}

Fig. 7 Structure of status appliance packet.

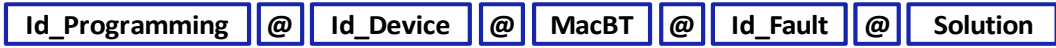

Fig. 8 Structure of Solution appliance packet.

If server receives a fault code for which there is no solution, the management application will mark the state of the failed programming ( $\mathrm{OK}=$ False). With this 
action, it avoids to rerun programming that generates a fault. The application will inform the user that he/she should contact to the customer support as soon as possible.

\section{Test bench and Operation system}

This section presents the graphic user interface developed for managing all appliances. We have also performed a network performance to analyze the data traffic in Bluetooth network.

\subsection{Graphic User Interface}

To manage, view and send the necessary orders to the different appliances, a graphic user interface has been developed using android. Figure 9 shows the main screens of our application. The first one allows users controlling programs of each appliance, as well as turns on/off and put it on hold. The second and third screens show a general view of house status. On the one hand, the second one shows the power consumption in watts and economic cost depending on the type of service contract and the current price set by the state or company. Finally, the third screen inform the user about the overall state of the system and if there is a problem with the supply of water or electricity. If a problem is detected, the system will perform various verification tasks in order to generate the correct orders.
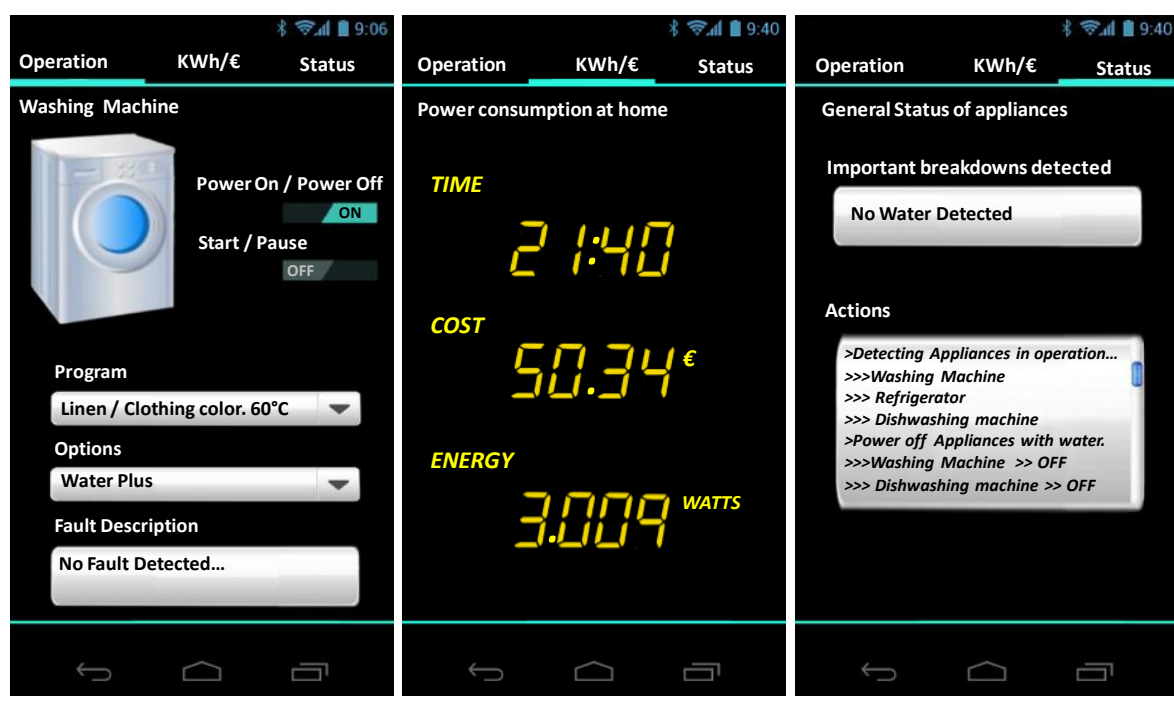

Fig. 9 Main Screens of control application.

With the selected information, the database is updated and the different schedules are sent to the appropriate device. The communication between the application and the different devices is done through sockets. 
The server only works with requests from one device although it may be simultaneously processing information from other devices.

\subsection{Test Bench}

The last step to ensure the correct operation of our system is to check the connectivity and operation of our network. In this subsection, we are going to perform a test bench with an appliance and our server. To perform our test, we have used a washing machine provided with a Bluetooth card and a mobile phone as a server. Figure 10 shows the scenario used in this test.

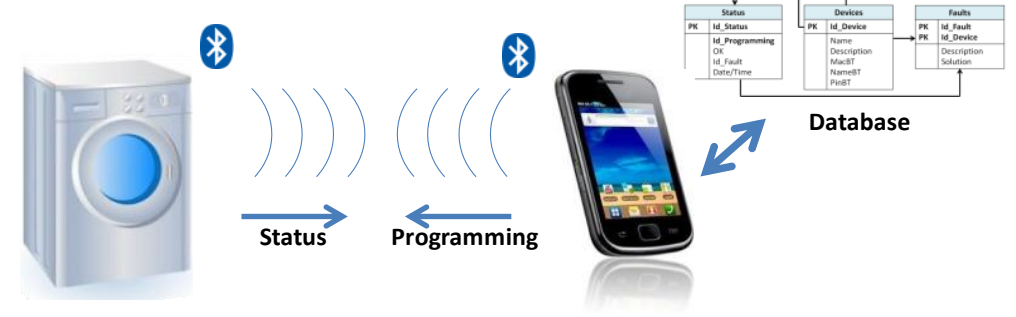

Fig. 20. Scenario used in the test bench.

The appliance periodically connects to the server. This communication permits the updating of status and programming in the database. The modifications on the database are done through SQL statements. Figure 11 shows the flow diagram of messages between an appliance, the server and the database.

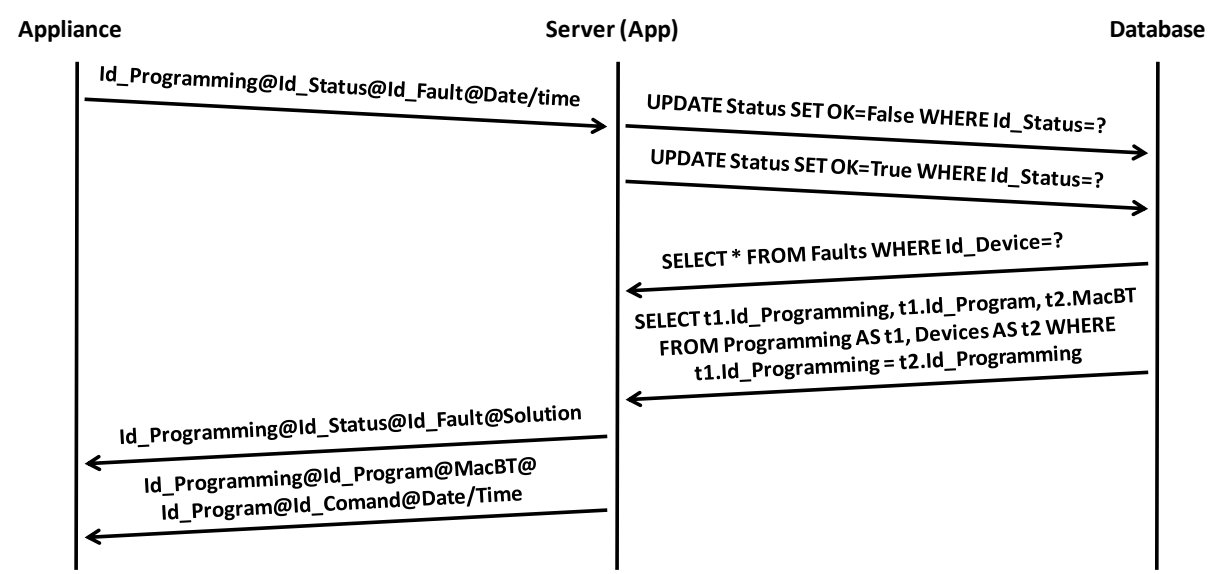

Fig. 31. Message diagram between the database, server App and appliance.

To perform our test, we have established a connection between the device and the mobile phone. The mobile phone simultaneously runs the control application and a 
network sniffer in order to capture the packets traffic generated. Figure 12 shows the total packets per second recorded in the communication between both devices.

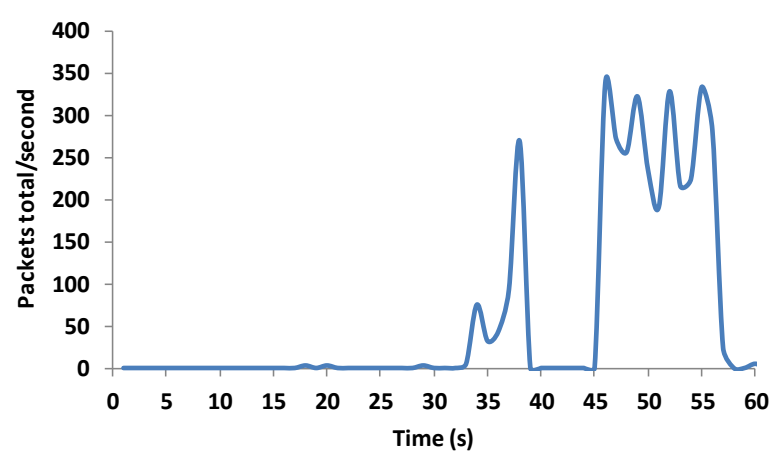

Fig. 42. Packets Total/second

Figure 13 shows the total bytes per second recorded in the communication between both devices.

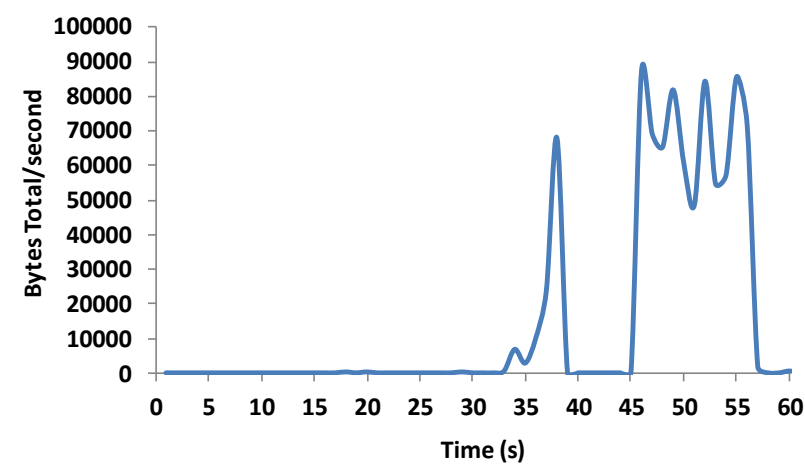

Fig. 53. Bytes Total/second

Figure 14 shows the bytes read per second recorded in the communication by server. As we can see the maximum volume of registered data is around 820 bytes.

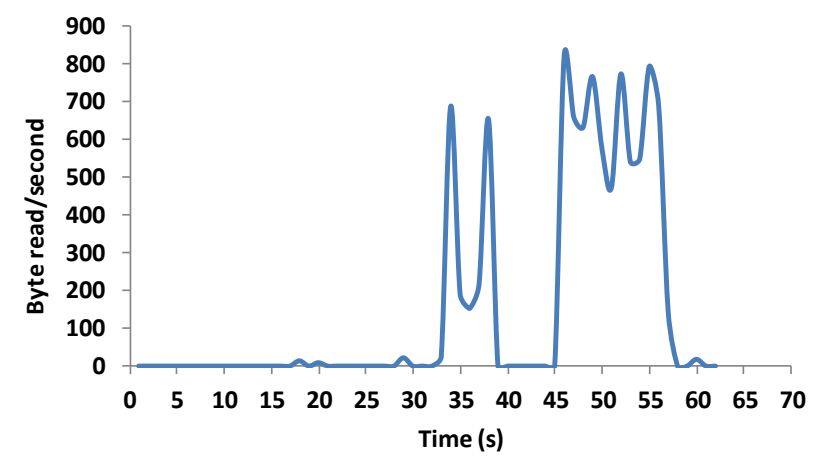

Fig. 64. Bytes read/second 
Figure 15 shows the bytes written per second recorded in the communication by server. In this case, the maximum volume of registered data is around 9000 bytes.

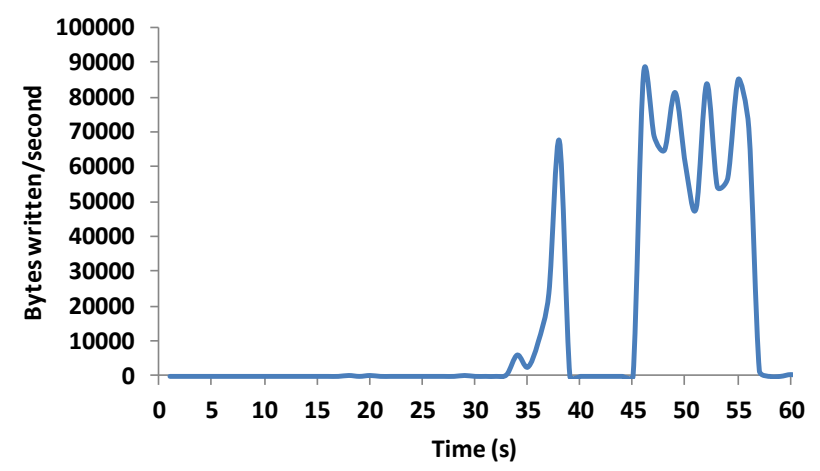

Fig. 75. Bytes written/second

The difference between bytes written and bytes read is because in this communication, the server is sending the different programming to the appliance and the appliance only sends information of its status.

Figures 12, 13, 14 and 15 show two zones with more data volume. The first of these zones is located between the $33^{\text {rd }}$ and $40^{\text {th }}$ second. In this period, the connection between devices is performed. The second period with big data volume is located between $45^{\text {th }}$ and $55^{\text {th }}$ second. During this interval, devices perform the transmission of information.

\section{Conclusion}

In this paper, we have presented a smart application for controlling appliances in home environments. Our application allows us monitoring and controlling, in real time, all of our appliances placed at home. The application has been implemented using Android, but we could develop this system for other mobile platforms.

The main advantage of this system is its versatility with any type of device, because we only need to provide each device with a Bluetooth cards. We can do it in different ways. First, there already have appliances with Bluetooth systems that can be used to connect with them through the server. However, for those devices that do not have this technology, we can use economic hardware such as Arduino and its complements that will allow us integrating this wireless technology with our appliances. The user interface is very simple and through it, it is very easy to control the power consumption at home. In addition, the smart algorithm is able to decide about which appliances should work as a function of the time and the home power consumption. This is possible due to the previous training period that system performs.

Our system allows us maintaining a stable energy and water consumption in our house. The system can also make use of reduced rates of light provider companies that will generate a considerable economic savings. 
As future works, we want to develop a secure system to integrate it to our programs in order to avoid any kind of threat [20] and vulnerability that can endanger our system and the stored data.

\section{References}

1. Garcia, M., Sendra, S., Lloret, J. and Canovas. A., Saving energy and improving communications using cooperative group-based Wireless Sensor Networks, Telecommunication Systems, 2013, Vol. 52, No. 4, pp 2489-2502.

2. Liu, Y. and Zhou, G. Technologies and Applications of Internet of Things. In Proceedings of 2012 Fifth International Conference on Intelligent Computation Technology and Automation (ICICTA), Zhangjiajie, China, 12-14 January 2012. Pp. 197-200.

3. Aiello, M. The Role of Web Services at Home. In Proceedings of the Advanced International Conference on Telecommunications and International Conference on Internet and Web Applications and Services, (AICT-ICIW'06), Guadeloupe, France, 23-25 February 2006.

4. Mowafi, M.Y., Awad, F.H., Al-Batati, M. A., Opportunistic Network Coding for Real-Time Transmission over Wireless Networks, Network Protocols and Algorithms, 2013, Vol. 5, No. 1, Pp. 1-19.

5. Gangadhar, G.; Nayak, S.; Puttamadappa, C. Intelligent Refrigerator with monitoring capability through internet. International Journal of Computer Applications. Special Issue on "Wireless Information Networks \& Business Information System, 2011, Vol. 2, No. 7, Pp. 65-68.

6. Soucek, S.; Russ, G.; Tamarit, C. The Smart Kitchen Project-An Application of Fieldbus Technology to Domotics. In Proceedings of 2nd International Workshop on Networked Appliances (IWNA2000), New Brunswick, NJ, USA, 30 November-1 December 2000.

7. Zhang, W., Tan, G-Z., and Ding N. Traffic Information Detection Based on Scattered Sensor Data: Model and Algorithms, Adhoc \& Sensor Wireless Networks, 2013, Vol 18, No.3-4, pp. $225-240$

8 Ranjit, J. S., and Shin, S. A Modified IEEE 802.15. 4 Superframe Structure for Guaranteed Emergency Handling in Wireless Body Area Network. Network Protocols \& Algorithms, 2013, Vol. 5, No. 2, Pp. 1-15.

9. Braeken, A. and Singelee, D. Efficient and Location-Private Communication Protocols for WBSNs, Adhoc \& Sensor Wireless Networks, 2013, Vol. 19, No.3-4, pp. 305-326

10. Augusto, J. C., McCullagh, P., McClelland, V., and Walkden, J. A. Enhanced healthcare provision through assisted decision-making in a smart home environment. In Proceedings of the Second Workshop on Artificial Intelligence Techniques for Ambient Intelligence (AITAmI'07), Hyderabad, India, January 6-7, 2007

11. Zhang, L., Zhao, Z., Li, D., Liu, Q. and Cui, L. Wildlife Monitoring Using Heterogeneous Wireless Communication Network, Adhoc \& Sensor Wireless Networks, 2013, Vol 18, No.3-4, pp. 159-179

12. Viani, F., Robol, F., Polo, A., Rocca, P., Oliveri, G., and Massa, A. Wireless Architectures for Heterogeneous Sensing in Smart Home Applications: Concepts and Real Implementation, Proceedings of the IEEE, 2013, Vol.101, No.11, pp.2381-2396

13. Lloret, J., Macías, E., Suárez, A., and Lacuesta, R. Ubiquitous Monitoring of Electrical Household Appliances. Sensors, 2012, Vol. 12, No.11, Pp. 15159-15191.

14. Kamilaris, A.; Trifa, V.; Pitsillides, A. The smart home meets the Web of Things International Journal of Ad Hoc and Ubiquitous Computing, 2011, Vol. 7, No. 3, Pp.145154 . 
15. Kamilaris, A., Trifa, V. and Pitsillides, A. HomeWeb: An Application Framework for WebBased Smart Homes. In Proceedings of the 18th International Conference on Telecommunications (ICT 2011), Ayia Napa, Cyprus, 8-11 May 2011; pp. 134-139.

16. IEEE Std 802.15.1-2002 - IEEE Standard for Information technology Telecommunications and information exchange between systems - Local and metropolitan area networks - Specific requirements Part 15.1: Wireless Medium Access Control (MAC) and Physical Layer (PHY) Specifications for Wireless Personal Area Networks (WPANs).

17. KNX international Site. [On line]. Available at: http://www.knx.org/knx-en/index.php. [Last access: February 1, 2014]

18. LonWorks Technology. [On line]. In ECHELON web site. Available at: http://www.echelon.com/technology/lonworks/ [Last access: February 1, 2014]

19. X10 protocol. [On line]. In X10 web site. Available at: http://X10-lang.org/ [Last access: February 1, 2014]

20. Rohini Basak, R. and Sardar, B., Security in Network Mobility (NEMO): Issues, Solutions, Classification, Evaluation, and Future Research Directions, Network Protocols and Algorithms, 2013, Vol. 5, No. 2, pp. 87-111. 